\title{
Prevalencia de disfunción eyaculatoria secundaria al tratamiento con alfa-bloqueantes en pacientes con hiperplasia benigna de próstata
}

\author{
Martín-Morales A*, Meyer G**, Ramírez E*** \\ *Servicio de Urología. Hospital Carlos Haya: **Sanofi-Aventis SA. ***Infociencia SL.
}

Actas Urol Esp. 2008;32(7):705-712

\begin{abstract}
RESUMEN
PREVALENCIA DE DISFUNCIÓN EYACULATORIA SECUNDARIA AL TRATAMIENTO CON ALFA-BLOQUEANTES EN PACIENTES CON HIPERPLASIA BENIGNA DE PRÓSTATA

Introducción: La hiperplasia benigna de próstata (HBP) se acompaña de sintomas del tracto urinario inferior (STUI) que pueden causar alteraciones eyaculatorias, aunque éstas podrian ser además consecuencia del tratamiento con alfa-bloqueantes.

Objetivos: Determinar la prevalencia de disfunción eyaculatoria en pacientes con HBP tratados con alfa-bloqueantes. Secundariamente, evaluar el efecto del tipo de fármaco y la relación de las alteraciones eyaculatorias con factores pronósticos y la gravedad de la HBP.

Material y métodos: Estudio epidemiológico, transversal, no controlado y abierto en 1.177 pacientes $\geq 40$ años diagnosticados de HBP/STUI con más de 6 meses de evolución, tratados con el mismo alfa-bloqueante durante como mínimo los últimos 3 meses. El grado de disfunción eyaculatoria se determinó mediante el dominio sobre eyaculación del Male Sexual Health Questionnaire (MSHQ). Los resultados se estratificaron según la edad y gravedad de la HBP, establecida mediante el International Prostate Symptoms Score (IPSS).

Resultados: Se ha estimado una prevalencia de disfunción eyaculatoria del $82,6 \%$ en pacientes con HBP/STUI en tratamiento con alfa-bloqueantes. Aunque generalmente leve, un amplio porcentaje de quienes la sufren la consideran un problema. La edad avanzada es el factor más influyente en la gravedad tanto de las alteraciones eyaculatorias como de los síntomas prostáticos. Entre ambos parámetros se ha establecido una sólida correlación. Finalmente, entre los alfa-bloqueantes evaluados, la alfuzosina se ha asociado a una mejor función sexual.

Conclusiones: Más del 80\% de pacientes con HBP sufren alteraciones eyaculatorias, estrechamente relacionadas con la severidad de los síntomas prostáticos y la edad del individuo. Al establecer tratamiento con alfa-bloqueantes, deberíamos tener en cuenta que la alfuzosina es el que menos impacto tiene sobre la función eyaculatoria.
\end{abstract}

Palabras clave: Hiperplasia benigna de próstata. Alfuzosina. Alfa-bloqueantes. Disfunción eréctil. Enfermedad urológica. Síntomas prostáticos. Tracto urinario inferior.

\begin{abstract}
PREVALENCE OF EJACULATORY DYSFUNCTION SECONDARY TO ALPHA-BLOCKER THERAPY IN PATIENTS WITH BENIGN PROSTATIC HYPERPLASIA

Introduction: Benign prostatic hyperplasia (BPH) is characterized by lower urinary tract symptoms (LUTS) that may cause ejaculatory disorders, although they could be also a consequence of alpha-blocker treatment.

Objectives: To determine the prevalence of ejaculatory dysfunction in patients with HBP undergoing alpha-blocker therapy. Secondary objectives were to evaluate the effect of different drugs on normal ejaculation and the relationship of dysfunction degree to prognostic factors and BPH severity.

Materials and methods: Epidemiologic, transversal, uncontrolled and open-label study in 1.177 patients older than 40, who had been diagnosed of BPH/LUTS with more than 6 months of evolution and treated with the same alpha-blocker for at least the last 3 months. Severity of ejaculatory dysfunction was determined by the domain on ejaculation of the Male Sexual Health Questionnaire (MSHQ). Results were stratified by age and BPH severity, determined by the International Prostate Symptoms Score (IPSS).

Results: The prevalence of ejaculatory dysfunction has been estimated to be $82.6 \%$ in patients with BPH/LUTS treated with alpha-blockers. Although usually mild, the dysfunction is considered as bothersome by a high percentage of those who suffer it. Advanced age is the most influential factor in the severity of both ejaculatory abnormalities and prostate symptoms. Moreover, a solid correlation between these two parameters has been established. Finally, among the analyzed alpha-blockers, alfuzosin has been associated with the best sexual function.

Conclussions: More than $80 \%$ of patients with BPH suffer ejaculatory abnormalities, which are closely related to the severity of prostate symptoms and increased age. When initiating alpha-blocker treatment, we should consider that alfuzosin is the one with less negative impact on ejaculatory function.
\end{abstract}

Keywords: Adrenergic alpha-Antagonists. Ejaculation. Prostatic Hyperplasia. Sexual Dysfunction, Physiological. Urologic Diseases. 
$\mathrm{L}$ a hiperplasia benigna de próstata (HBP) afecta en España al 50-80\% de la población masculina entre los 50 y 80 años $^{1}$. A los 60 , la mitad de los varones presentan ya signos microscópicos de HBP y a los 70 , más del $40 \%$ tienen un aumento del tamaño de la próstata detectable en una exploración rutinaria.

Este aumento se acompaña de síntomas del tracto urinario inferior (STUI), tanto obstructivos (dificultad y retraso en la micción, goteo post-miccional, sensación de vaciado incompleto) como irritativos (urgencia miccional, dolor suprapúbico, incontinencia). Los pacientes con síntomas leves o moderados, que no requieren cirugía, son candidatos a un tratamiento farmacológico ${ }^{2}$. Por su seguridad y efectividad, los bloqueantes de los receptores alfa- 1 son el tratamiento actual de primera elección. Estos fármacos reducen el tono simpático de la musculatura del cuello vesical y de la próstata, mejorando la sintomatología e incrementando el flujo urinario máximo sin modificar el tamaño prostático en un plazo de 2-3 semanas. Además, la especificidad por el receptor alfa de tipo 1, minimiza los efectos indeseables ya que no bloquean los receptores del músculo liso en otras localizaciones ${ }^{3}$. En concreto, la alfuzosina ha demostrado recientemente ser un bloqueador uroselectivo al producir beneficios clínicos sobre la obstrucción y los sintomas del tracto urinario inferior con una incidencia mínima de efectos secundarios $^{4-8}$. Incluso comparada con otros fármacos para el tratamiento de la HBP, ha resultado ser más efectiva que finasterida y que la combinación de ambas ${ }^{9}$ en evaluaciones a corto plazo.

La prevalencia y repercusión de la disfunción eyaculatoria en hombres con STUI sugestivo de HBP, ha sido estudiada en 5.096 pacientes de Europa, Asia, América Latina, Medio Oriente y Canadá, con la conclusión de que alrededor de un $20 \%$ experimentan dolor y molestias en la eyaculación ${ }^{10}$. Algunos estudios han sugerido una posible relación entre el tratamiento farmacológico y el incremento en el riesgo de disfunción eyaculatoria $^{11}$, mientras que otros trabajos han demostrado incluso una notable mejoría en un elevado porcentaje de los pacientes ${ }^{12}$. La gran controversia existente en este sentido justifica, y este es el objetivo, la necesidad de un estudio para determinar la prevalencia real de disfunción eyaculatoria asociada al empleo de alfa-bloqueantes en nuestro entorno, así como la relación entre los distintos tipos de fármacos y la aparición de estos síntomas. Para ello, se ha utilizado el Cuestionario IPSS ${ }^{13}$ (International Prostate Symptoms Score - Puntuación Internacional de los Síntomas Prostáticos), promovido por la OMS para evaluar y medir los síntomas de la HBP/STUI, y el dominio de la disfunción eyaculatoria del Cuestionario sobre la Salud Sexual Masculina (MSHQ) ${ }^{14}$.

\section{MATERIAL Y MÉTODOS}

\section{Diseño y pacientes}

Se trata de un estudio epidemiológico, transversal, no controlado, abierto, sobre un conjunto de pacientes atendidos en Servicios de Urología/Andrología españoles, de edad $\geq 40$ años, con HBP y STUI con al menos 6 meses de evolución, y tratados con el mismo tipo de alfa-bloqueante durante, como mínimo, los últimos 3 meses.

Fueron excluidos del estudio los pacientes con antecedentes de tratamientos invasivos para la HBP; los que tenían antecedentes de neoplasia urológica; los que presentaran alteraciones neurológicas, cirugía pélvica, traumatismo pélvico, diabetes mellitus mal controlada o neuropatía diabética; aquellos con ausencia de datos en la historia clínica; o los que sufrieran cualquier trastorno que los incapacitara para cumplimentar los formularios.

El tamaño de muestra se calculó en 1.200 pacientes, por lo que cada uno de los 400 investigadores previstos debía incluir 3 pacientes. Se efectuó una única visita en la que se recopilaron datos clínicos y de la historia clínica del paciente, y se siguieron en todo momento los procedimientos habituales de la práctica clínica. Además, el estudio se desarrolló de acuerdo con los principios de la Declaración de Helsinki.

\section{Variables}

Con el objetivo de conocer la prevalencia de la disfunción eyaculatoria en pacientes con HBP/STUI tratados con alfa-bloqueantes, se obtuvo como variable principal la puntuación del Cuestionario MSHQ. Otros objetivos planteados incluyeron evaluar los efectos del tipo de alfa-bloqueante sobre la función eyaculatoria, la posible influencia de los factores pronósticos (edad, ocupación, etc.) en el grado de las alteraciones, y la relación entre el grado de la disfunción y la gravedad de la HBP. Por lo tanto, como variables secundarias se recogieron la pun- 
tuación del Formulario IPSS, el tipo de alfa-bloqueante empleado y las posibles patologías y tratamientos concomitantes.

Se establecieron 3 categorías para la severidad de la HBP en base a la puntuación del IPSS (leve: 07, moderada: 8-19, severa: 20-35). Para clasificar la sintomatología de la función eyaculatoria, y debido a la falta de referencias previas, se extrapolaron los mismos rangos a la escala $\mathrm{MSHQ}$, definiendo 4 categorías (normal: 37-40, leve: 29-36, moderada: 1728, severa: 0-16). Los resultados de las alteraciones en la función eyaculatoria se estratificaron en función de la edad y de la gravedad de la HBP.

\section{Análisis estadístico}

Todas las variables registradas se analizaron mediante métodos descriptivos. Se generaron tablas de frecuencias absolutas y relativas para las variables cualitativas, y tablas de estadísticos (medidas de tendencia central y dispersión) para las variables cuantitativas. La posible relación entre las variables categóricas se analizó mediante la prueba Chi cuadrado. Se comprobó la normalidad de los datos recogidos mediante el test de Kolmogorov-Smirnov. En el caso de variables continuas y normales, se utilizó el test t-Student, y en caso de datos no normales, se emplearon pruebas no paramétricas (prueba de Wilcoxon o prueba de los signos). Para establecer la influencia de distintos factores pronósticos sobre la variable principal, se realizó una regresión logística y/o lineal. Para todos los análisis se estableció un intervalo de confianza del 95\%.

\section{RESULTADOS \\ Descriptiva de la población}

El estudio DESEA ha incluido una muestra de 1.177 pacientes evaluables con HBP/STUI, tratados con alfa-bloqueantes. Las variables demográficas, antropológicas y socioeconómicas, se describen en la Tabla 1.

En la práctica totalidad de los pacientes, la HBP había sido diagnosticada mediante tacto rectal y ecografía. Los parámetros de la enfermedad y de la sintomatología del tracto urinario inferior se incluyen en la Tabla 2, junto a los datos relativos a tratamientos y enfermedades concomitantes.

Los alfa-bloqueantes fueron el tipo de fármaco mayoritariamente usado para tratar la HBP, tanto entre los tratamientos previos al evaluado en el estu-
Tabla 1. Datos demográficos, antropológicos y socioeconómicos de los participantes

\begin{tabular}{|c|c|c|}
\hline Variable & & Total \\
\hline Edad (años) & Media (DE) & $64,31(8,17)$ \\
\hline \multirow[t]{3}{*}{ Hábitat } & Rural (<2.000 hab.) & $184(16,6 \%)$ \\
\hline & $\begin{array}{l}\text { Intermedio } \\
(2.000-10.000 \text { hab.) }\end{array}$ & $245(22,1 \%)$ \\
\hline & Urbano (>10.000 hab.) & $682(61,4 \%)$ \\
\hline Peso (kg) & Media (DE) & $79,40(9,99)$ \\
\hline Talla (cm) & Media (DE) & $170,30(6,39)$ \\
\hline IMC $\left(\mathrm{kg} / \mathrm{m}^{2}\right)$ & $\begin{array}{l}\text { Bajo y normopeso } \\
\text { (IMC }<25) \\
\text { Obesidad grado } \\
\text { I }(25 \leq \text { IMC }<30) \\
\text { Obesidad grado II } \\
\text { (30 } \leq \text { IMC }<35 \text { ) } \\
\text { Obesidad grado III } \\
(35 \leq \text { IMC }<40) \\
\text { Obesidad grado IV } \\
(40 \leq \text { IMC) }\end{array}$ & $\begin{array}{c}206(18,8 \%) \\
716(65,2 \%) \\
158(14,4 \%) \\
13(1,2 \%) \\
5(0,5 \%)\end{array}$ \\
\hline PAS (mm Hg) & Media (DE) & $138,84(14,57)$ \\
\hline $\mathrm{PAD}(\mathrm{mm} \mathrm{Hg})$ & Media (DE) & $81,44(10,85)$ \\
\hline $\begin{array}{l}\text { Frecuencia } \\
\text { cardiaca } \\
\text { (latidos/min) }\end{array}$ & Media (DE) & $74,79(9,31)$ \\
\hline $\begin{array}{l}\text { Ocupación } \\
\text { laboral }\end{array}$ & $\begin{array}{l}\text { Activo } \\
\text { En paro } \\
\text { Invalidez transitoria } \\
\text { Invalidez total } \\
\text { Jubilado }\end{array}$ & $\begin{array}{c}517(46,3 \%) \\
8(0,7 \%) \\
9(0,8 \%) \\
13(1,2 \%) \\
569(51,0 \%)\end{array}$ \\
\hline Entorno familiar & $\begin{array}{l}\text { Vive solo } \\
\text { Vive en familia } \\
\text { Institucionalizado }\end{array}$ & $\begin{array}{c}105(9,4 \%) \\
1009(90,4 \%) \\
2(0,2 \%)\end{array}$ \\
\hline $\begin{array}{l}\text { Nivel de } \\
\text { escolarización }\end{array}$ & $\begin{array}{l}\text { Sin estudios } \\
\text { Elemental } \\
\text { Medios } \\
\text { Superior/Universitario }\end{array}$ & $\begin{array}{c}95(8,5 \%) \\
489(43,8 \%) \\
393(35,2 \%) \\
139(12,5 \%)\end{array}$ \\
\hline
\end{tabular}

dio, como entre los tomados durante 3 meses previos a la visita de inclusión (Tabla 2). El 38,0\% de los pacientes recibían además medicación para tratar patologías concomitantes, presentes en el 60,3\% de la población en estudio. Más de un tercio de los individuos eran hipertensos, por lo que destaca el elevado uso de fármacos antihipertensivos. Los más comunes fueron los IECAs y los diuréticos, representando respectivamente un $44,0 \%$ y un $26,4 \%$ del total de tratamientos concomitantes. Además, un 26,2\% de los pacientes presentaron otras medicaciones no listadas en la Tabla 2. 
Tabla 2. Antecedentes médicos de los participantes

\begin{tabular}{|c|c|c|}
\hline Variable & & Total $^{1}$ \\
\hline Antigüedad de la enfermedad (años) & Media (DE) & $3,61(3,21)$ \\
\hline Volumen de la próstata (ml) & Media (DE) & $50,80(19,63)$ \\
\hline Gravedad clínica de la $\mathrm{HBP}^{2}$ & $\begin{array}{l}\text { Leve } \\
\text { Moderada } \\
\text { Severa }\end{array}$ & $\begin{array}{c}320(28,7 \%) \\
735(65,9 \%) \\
60(5,4 \%)\end{array}$ \\
\hline Valor del PSA (ng/ml) & Mediana & $2,7^{3}$ \\
\hline Retención aguda de orina & Sí & $44(4,0 \%)$ \\
\hline Tratamiento quirúrgico previo ${ }^{4}$ & Sí & $110(9,8 \%)$ \\
\hline Tratamiento farmacológico previo para HBP & $\begin{array}{l}\text { Alfa-bloqueantes } \\
\text { Extractos vegetales } \\
\text { Inhibidores de la 5-alfa-reductasa }\end{array}$ & $\begin{array}{c}628(56,4 \%) \\
288(25,9 \%) \\
139(8,8 \%)\end{array}$ \\
\hline Alfa-bloqueantes en los últimos 3 meses & $\begin{array}{l}\text { Alfuzosina } \\
\text { Tamsulosina } \\
\text { Terazosina } \\
\text { Doxazosina }\end{array}$ & $\begin{array}{c}575(53,3 \%) \\
339(31,4 \%) \\
82(7,6 \%) \\
82(7,6 \%)\end{array}$ \\
\hline Patologías concomitantes & $\begin{array}{l}\text { Hipertensión arterial } \\
\text { Dislipemia } \\
\text { Diabetes mellitus } \\
\text { Patología respiratoria crónica } \\
\text { Patología reumática } \\
\text { Cardiopatía isquémica } \\
\text { Otras patologías }\end{array}$ & $\begin{array}{c}390(34,9 \%) \\
235(21,0 \%) \\
98(8,8 \%) \\
82(7,3 \%) \\
78(7,0 \%) \\
54(4,8 \%) \\
71(6,4 \%)\end{array}$ \\
\hline Medicaciones concomitantes & $\begin{array}{l}\text { IECAs } \\
\text { Diuréticos } \\
\text { ARA-II } \\
\text { Beta-bloqueantes } \\
\text { Calcio-antagonistas } \\
\text { Antihipertensivos centrales } \\
\text { Nitratos y derivados } \\
\text { Inhibidores de la PDES }\end{array}$ & $\begin{array}{c}187(16,7 \%) \\
112(10,0 \%) \\
56(5,0 \%) \\
53(4,8 \%) \\
52(4,7 \%) \\
41(3,7 \%) \\
21(1,9 \%) \\
19(1,7 \%)\end{array}$ \\
\hline
\end{tabular}

${ }^{1}$ Porcentajes calculados sobre el total de la población evaluable.

${ }^{2}$ Severidad de la enfermedad estimada por el investigador.

${ }^{3}$ Se muestra la mediana porque la media y la DE están influidas por un valor máximo de PSA de $270 \mathrm{ng} / \mathrm{ml}$.

${ }^{4}$ De cualquier órgano y sistema, antes de la inclusión en el estudio.

\section{Sintomas prostáticos y disfunción eyaculatoria (IPSS y MSHQ)}

Los resultados obtenidos revelaron una puntuación media de $13,48 \pm 6,02$ sobre un máximo de 35 . La clasificación de la población en estudio en categorías según esta puntuación reflejó que el 66,7\% de los individuos tenían una sintomatología moderada, mientras que las sintomatologías leve y severa se presentaron en un 16,7\% cada una. Esta clasificación correlacionó de forma altamente significativa con la gravedad clínica de la HBP estimada por el médico ( $\mathrm{p}<0,0001)$. De este modo, los pacientes con síntomas leves según el IPSS, presentaron mayoritariamente $(72,4 \%)$ HBP clínicamente leve según el criterio del médicoy en ningún caso severa, mientras que aquellos con sintomas pros- táticos severos presentaron, en general $(96,1 \%)$, HBP clínicamente moderada o severa a juicio del médico. Con respecto a la calidad de vida derivada de los síntomas prostáticos, el porcentaje de sujetos satisfechos $(36,6 \%)$ e insatisfechos $(35,0 \%)$ fue muy similar.

Asimismo, el 82,6\% de los pacientes presentaron algún tipo de disfunción eyaculatoria según las puntuaciones del Cuestionario MSHQ, siendo la disfunción leve, en la mitad de los casos (51,9\%), moderada en el 27,3\% de ellos, y severa en tan sólo el 3,4\%. El valor promedio del MSHQ fue de 30,70 $\pm 6,46$. La distribución de puntuaciones de los cuestionarios IPSS y MSHQ, que evalúan respectivamente la gravedad de la sintomatología prostática y de las alteraciones eyaculatorias, se muestra en la Figura 1. 


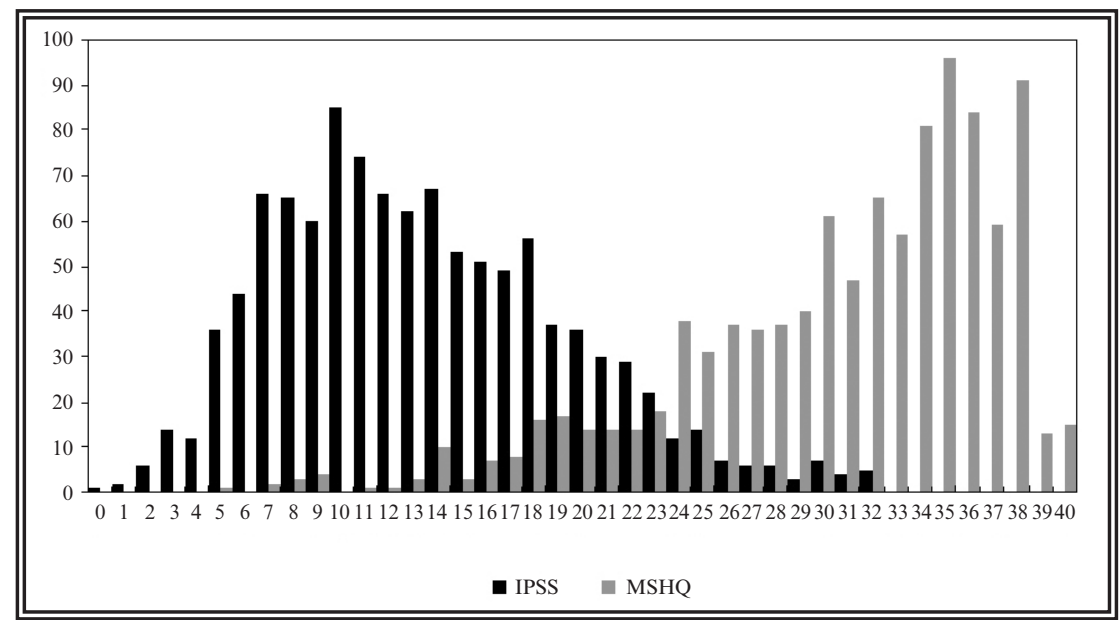

FIGURA 1. Comparación de la distribución de puntuaciones MSHQ e IPSS.

Las categorias de sintomatologia prostática y disfunción eyaculatoria presentan una distribución simétrica. La puntuación máxima del cuestionario IPSS es de 35 puntos (mayor gravedad de los sintomas prostáticos), mientras que la del MSHQ se sitúa en 40 (mínima alteración eyaculatoria), por lo que esta representación es aproximada.

Se observaron diferencias altamente significativas $(p<0,0001)$, tanto en la severidad de la HBP/ STUI como en la prevalencia y severidad de las alteraciones eyaculatorias, en función de la edad de los pacientes (Fig. 2). Así, en edades más tempranas,

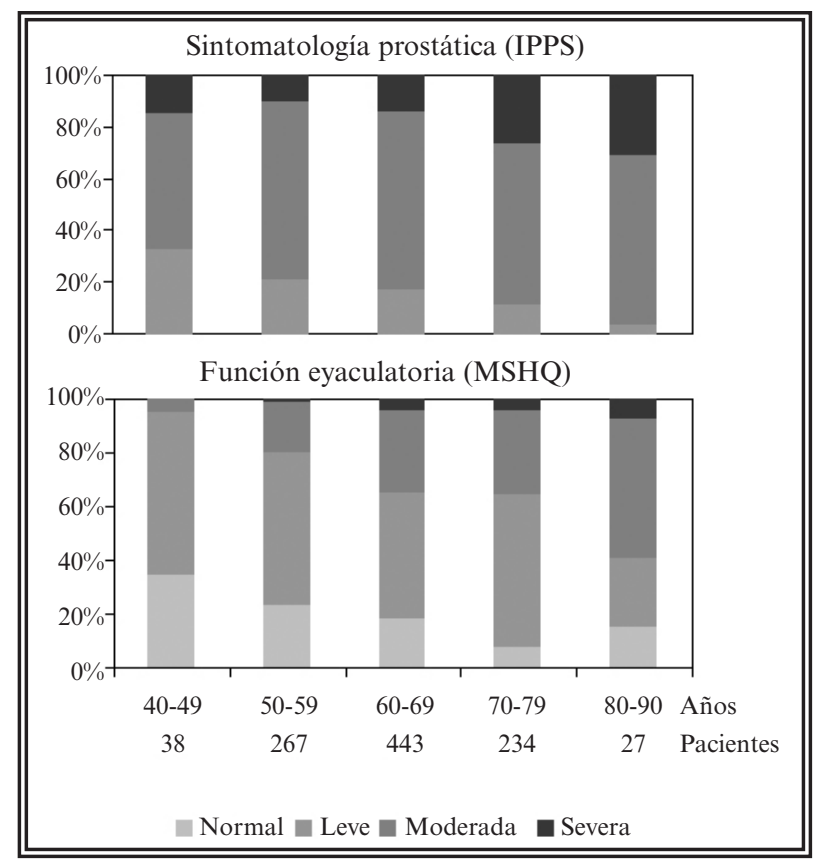

FIGURA 2. Sintomatología prostática y disfunción eyaculatoria según la edad.

Tanto la distribución de la gravedad de los sintomas prostáticos (calculada según la puntuación IPSS), como de la severidad de las alteraciones los grados de las alteraciones en la función eyaculatoria (según el test MSHQ), presentaron diferencias significativas según los grupos de edad ( $p<0,0001$ en la prueba Chi cuadrado). los síntomas prostáticos leves predominaban sobre los severos $(32,5 \%$ vs. $15,0 \%$, en pacientes de 40-49 años), mientras que estas proporciones se invertían en edades más avanzadas $(3,1 \%$ vs. $31,3 \%$, en pacientes de 80-90 años). Del mismo modo, la prevalencia de las alteraciones eyaculatorias se incrementaba con la edad $(65,8 \%$ a los $40-49$ años vs. $85,2 \%$ a los $80-90$ ), al igual que la gravedad de éstas. En un extremo se situaban los pacientes más jóvenes (40-49 años), que no presentaban disfunciones severas y eran, en su mayoría, casos leves $(60,5 \%)$ o asintomáticos $(34,2 \%)$, mientras que en el otro estaban los pacientes más seniles, con alteraciones generalmente moderadas $(51,9 \%)$ y un $7,4 \%$ de disfunciones severas.

Destaca, además, que la mitad de los pacientes $(46,1 \%)$ manifestaron sentirse molestos por el hecho de padecer problemas eyaculatorios. Esta pregunta presentó una elevada correlación con la puntuación obtenida en el resto de preguntas de función eyaculatoria del cuestionario MSHQ (correlación de Spearman: 0,647, $\mathrm{p}<0,0001)$.

\section{Tratamientos con alfa-bloqueantes}

Hay diferencias significativas $(\mathrm{p}<0,0001)$ en la disfunción eyaculatoria cuando se clasifican los pacientes en base al tratamiento alfabloquante. (Fig. 3). Al comparar los dos fármacos de uso más común, la alfuzosina y la tamsulosina, se aprecia que los pacientes tratados con alfuzosina presentan una función eyaculatoria normal en un $24,0 \%$ de los casos, una disfunción leve en el 56,6\% y sólo un 1,1\% padecen disfunciones severas. En cambio, los tratados con tamsulosina, presentan mayoritariamente disfunciones leves $(42,6 \%)$ y moderadas $(42,3 \%)$, y hasta un $7,2 \%$ de los casos sufren disfunciones severas. Diferencias similares, aunque más atenuadas, también se observan al comparar la alfuzosina con los otros dos fármacos, terazosina y doxazosina.

\section{Factores pronósticos}

Los resultados de los análisis de regresión entre los diversos factores pronósticos y el grado de las alteraciones eyaculatorias se presentan en la Tabla 3. 


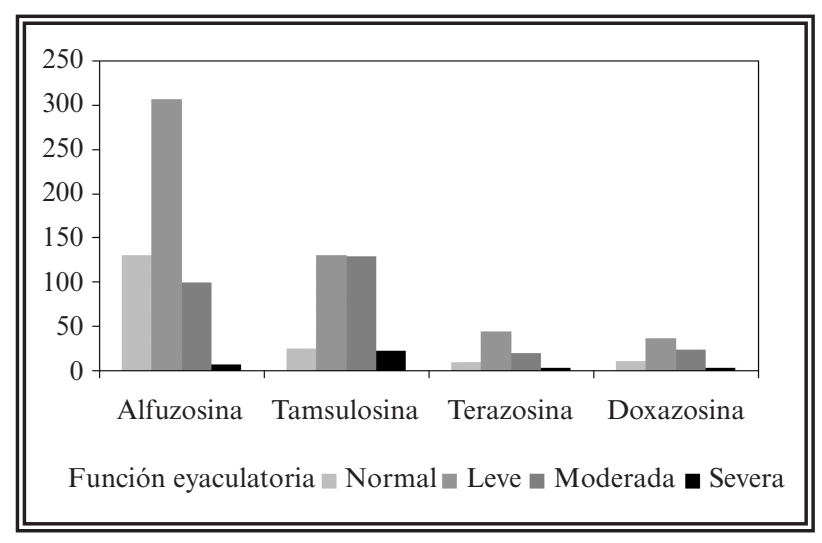

FIGURA 3. Relación entre los tratamientos alfa-bloqueantes y sus efectos sobre las alteraciones en la función eyaculatoria.

La distribución de la gravedad de la disfunción eyaculatoria presentó diferencias significativas según el tipo de tratamiento ( $p<0,0001$ en la prueba Chi cuadrado).

El análisis univariante corrobora el efecto de la edad antes mencionado, tanto en la gravedad de las disfunciones como en la prevalencia de las mismas. Por otro lado, el nivel de escolarización también parece influir en la gravedad de las disfunciones eyaculatorias. Así, encontramos diferencias significativas entre pacientes con estudios superiores y pacientes con estudios elementales ( $\mathrm{p}=0,0004)$, de modo que los primeros parecen tener un menor riesgo de sufrir disfunciones eyaculatorias o las sufren en menor grave- dad $(\mathrm{OR}=0,44)$. Y lo mismo ocurre entre pacientes con estudios elementales y pacientes sin estudios ( $\mathrm{p}=0,0335$ y $\mathrm{OR}=1,52)$, aunque el mayor intervalo de confianza en este caso no permite establecer influencias más concluyentes (IC 95\%: 0,70-3,31).

Sin embargo, la regresión multivariante muestra que el único factor que influye significativamente en el grado de alteración de la función eyaculatoria es la edad, $(\mathrm{p}<0,0001)$ con un efecto relativamente pequeño $(\mathrm{OR}=1,064)$ pero con un intervalo de confianza muy consistente (IC 95\%:1,04-1,09). Por el contrario, el nivel de escolarización no muestra influencias significativas sobre la gravedad de la disfunción.

\section{Correlación entre la disfunción eyaculatoria y la gravedad de la HBP}

Con el fin de evaluar la posible relación entre el grado de las alteraciones de la función eyaculatoria, estimado mediante el cuestionario MSHQ, y la gravedad clínica de la HBP, se realizó una correlación de Spearman. Este análisis revela una correlación fuertemente significativa entre estos dos parámetros, con un valor de 0,256 ( $\mathrm{p}<0,0001)$.

La prevalencia de disfunción eyaculatoria presenta diferencias significativas según la gravedad clínica de la HBP ( $p<0,0001)$. Casi la mitad de los pacientes sin alteraciones en la eyaculación presen-

Tabla 3. Regresión de los factores pronósticos y el grado de disfunción eyaculatoria

\begin{tabular}{|c|c|c|c|c|c|}
\hline \multicolumn{6}{|c|}{ REGRESIÓN UNIVARIANTE } \\
\hline Variable & Chi cuadrado & Efecto & Odds ratio & IC (95\%) & $\mathbf{N}$ \\
\hline Edad & $<0,0001$ & Edad & 1,0640 & $(1,04 ; 1,09)$ & 1009 \\
\hline IMC & 0,7119 & IMC & 1,0097 & $(0,96 ; 1,06)$ & 1011 \\
\hline Hábitat & $\begin{array}{l}0,8251 \\
0,2674\end{array}$ & $\begin{array}{l}\text { Urbano vs. Rural } \\
\text { Intermedio vs. Rural }\end{array}$ & $\begin{array}{l}0,8147 \\
0,7169\end{array}$ & $\begin{array}{l}(0,50 ; 1,32) \\
(0,42 ; 1,23)\end{array}$ & 1019 \\
\hline Ocupación & $\begin{array}{l}0,9632 \\
0,9626 \\
0,9600\end{array}$ & $\begin{array}{l}\text { Jubilado vs. Activo } \\
\text { Invalidez total vs. Activo } \\
\text { En paro vs. Activo }\end{array}$ & $\begin{array}{l}2,6870 \\
2,6027 \\
2,2773\end{array}$ & $\begin{array}{l}(1,04 ; 1,09) \\
(0,32 ; 21,0) \\
(0,28 ; 18,7)\end{array}$ & 1023 \\
\hline Entorno & 0,9789 & En familia vs. Solo & 0,7524 & $(0,41 ; 1,38)$ & 1024 \\
\hline Nivel escolar & $\begin{array}{l}0,0004 \\
0,0335 \\
0,2790\end{array}$ & $\begin{array}{c}\text { Superior/Univ. vs. Elemental } \\
\text { Sin estudios vs. Elemental } \\
\text { Medio vs. Elemental }\end{array}$ & $\begin{array}{l}0,4364 \\
1,5248 \\
0,7042\end{array}$ & $\begin{array}{l}(0,27 ; 0,70) \\
(0,70 ; 3,31) \\
(0,49 ; 1,02)\end{array}$ & 1023 \\
\hline \multicolumn{6}{|c|}{ REGRESIÓN MULTIVARIANTE } \\
\hline Variable & Estimación & Error estándar & Chi-cuadrado & Odds ratio & IC (95\%) \\
\hline Intercept & $-2,3450$ & 0,67 & 0,0005 & & \\
\hline Edad & 0,0620 & 0,01 & $<0,0001$ & 1,0640 & $(1,04 ; 1,09)$ \\
\hline
\end{tabular}


tan hipertrofia prostática leve $(45,2 \%)$ y muy raramente severa $(1,1 \%)$, mientras que los pacientes con algún tipo de alteración eyaculatoria presentan principalmente HBP moderada $(68,6 \%)$ y el porcentaje de hipertrofia severa aumenta hasta el 5,3\%.

Al subdividir los pacientes con disfunción eyaculatoria, según la gravedad de las alteraciones, la relación se mantiene $(\mathrm{p}<0,0001)$. La mayoría de los pacientes con HBP leve $(85,0 \%)$ no presentan disfunciones eyaculatorias o éstas son leves, mientras que los pacientes con hipertrofia severa presentan en más de la mitad de los casos $(57,4 \%)$ disfunciones eyaculatorias moderadas o severas. En términos generales, se observa que una mayor gravedad de la HBP implica una mayor gravedad de la disfunción eyaculatoria (Fig. 4).

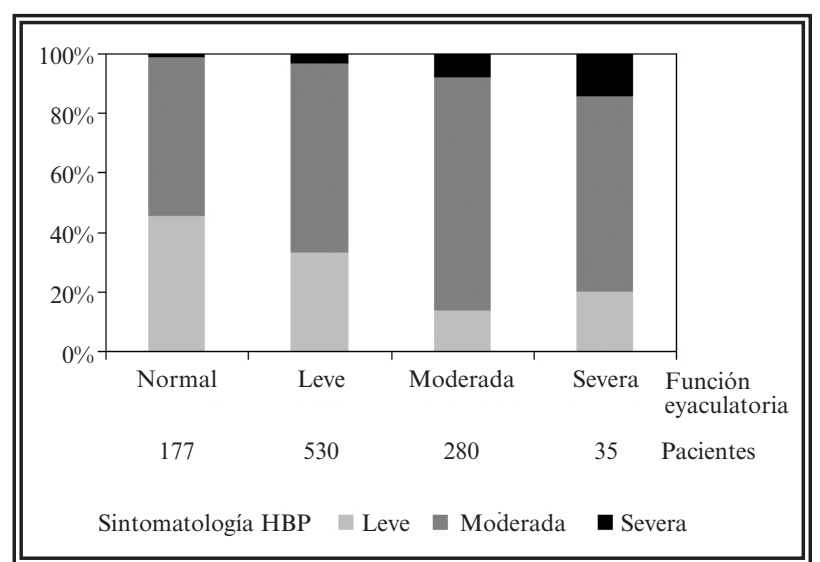

FIGURA 4. Relación entre la gravedad de los sintomas de la HBP/STUI y el grado de las alteraciones en la función eyaculatoria.

La sintomatologia de la hipertrofia prostática y las alteraciones en la función eyaculatoria están correlacionados de modo que una mayor severidad de la HBP implica un mayor gravedad de la disfunción eyaculatoria ( $<<0,0001$ en la prueba Chi cuadrado).

\section{DISCUSIóN}

En el presente estudio, se ha estimado una prevalencia del $82,6 \%$ de alteraciones en la función eyaculatoria en una población con HBP/STUI tratada con alfa-bloqueantes, mediante el dominio de la función eyaculatoria del cuestionario MSHQ, sobre salud sexual masculina. Predominan las alteraciones leves, observadas en dos tercios de los pacientes, mientras que las disfunciones severas se hallan en un $4 \%$ de los casos. Estas disfunciones se perciben de forma negativa por un amplio porcentaje de los sujetos que las padecen y por lo tanto deben ser tenidas en cuenta y discutidas a la hora de indicar un tratamiento.

El análisis de los diversos factores potencialmente pronósticos, ha revelado que la edad es el factor más influyente en la gravedad de los sintomas prostáticos y en la presencia y grado de las alteraciones en la función eyaculatoria. Así, a edades más avanzadas se observa una mayor severidad de la disfunción eyaculatoria, de acuerdo con las estadísticas univariante y multivariante. Sin embargo, comparaciones entre subgrupos según el nivel de escolarización han mostrado diferencias significativas en la regresión univariante, que podrían ser debidas al mayor grado de cumplimiento terapéutico por parte de los pacientes con un grado de formación más elevado.

Estudios realizados en todo el mundo indican que la presencia y gravedad de los síntomas prostáticos son factores de riesgo independiente en el desarrollo de trastornos eyaculatorios en edades avanzadas $^{15,16}$. En este sentido, nuestro estudio ha establecido, además, una relación sólida entre el grado de alteraciones en la función eyaculatoria y la gravedad de la HBP/STUI. Estos dos parámetros están correlacionados significativamente de modo que los pacientes con función eyaculatoria normal suelen presentar HBP más leve que los pacientes con algún tipo de disfunción. Además, los pacientes con una sintomatología prostática leve presentan principalmente $(85,0 \%)$ una función eyaculatoria normal o una disfunción leve, mientras los pacientes con HBP severa presentan el patrón inverso.

Finalmente, los alfa-bloqueantes, que constituyen la primera opción farmacológica actual para tratar la HBP/STUI, han mostrado un efecto sobre la función eyaculatoria dependiente del tipo de fármaco. Dentro de este grupo de fármacos, la alfuzosina se ha asociado claramente a una mejor función eyaculatoria en comparación con la tamsulosina, la terazosina y la doxazosina. Ya en un estudio anterior en pacientes con HBP/STUI y eyaculación dolorosa, el tratamiento con alfuzosina mejoró significativamente los sintomas prostáticos, las molestias eyaculatorias, el volumen eyaculado y, especialmente, la función eréctil ${ }^{17}$. Asimismo, otro estudio en voluntarios sanos demostró la superioridad de la alfuzosina frente a la tamsulosina en relación a la función sexual, con un menor número de casos de volumen eyaculado reducido y aneyaculación ${ }^{18}$. 


\section{CONCLUSIÓN}

A tenor de la información de estudios previos y la relación entre los distintos alfa-bloqueantes y la función eyaculatoria observada en este estudio, la alfuzosina resultaría el fármaco de elección en pacientes preocupados por mantener su función sexual, ya que además de presentar una buena tolerabilidad y elevada efectividad, es la menos relacionada con disfunción eyaculatoria.

\section{Agradecimientos}

Agradecemos la colaboración de todos los investigadores del estudio DESEA. Al Grupo Sanofi-Aventis SA por el patrocinio del estudio DESEA y a INFOCIENCIA SL por su colaboración en el análisis estadístico.

\section{REFERENCIAS}

1. Carballido J, Rodríguez JM, Del Llano JE. Hiperplasia prostática benigna y medicina basada en la evidencia. Su aproximación a la práctica clínica. Med Clin. 2000;114 Supl 2:96-104.

2. Jardin A, Bensadoun H, Delauche Cavallier M, Attali P. The BPH-ALF Group. Alfuzosin for treatment of benign prostatic hypertrophy. The BPH-ALF Group. Lancet 1991;337(8755): 1457-1461.

3. Lozano JA. Diagnostico y tratamiento de la hiperplasia benigna de próstata. Offarm. 2003;22(5):90-98.

4. Buzelin J, Fonteyne E, Kontturi M, Witjes W, Khan A. Comparison of Tamsulosin with Alfuzosin in the treatment of patients with lower urinary tract symptoms suggestive of bladder outlet obstruction (symptomatic benign prostatic hyperplasia). $\mathrm{Br} \mathrm{J}$ Urol. 1997;80(4):597-605.

5. Lukacs B, Grange JC, Comet D, McCarthy C. History of 7093 patients with coger urinary tract symptoms related to benign prostatic hyperplasia treated with Alfuzosin in general practice up to 3 years. Eur Urol. 2000;37(2):183-190.

6. Sánchez-Chapado M, Guil M, Alfaro V, Badiella L, FernándezHernando N. Safety and efficacy of sustained-released Alfuzosin on lower urinary tract symptoms suggestive of benign prostatic hyperplasiain 3095 Spanish patients evaluated during general practice. Eur Urol. 2000;37(4):421-427.

7. McNeill SA, Daruwala PD, Mitchell ID, Shearer MG, Hargreave TB. Sustained-release Alfuzosin and trial without catheter after acute urinary retention: a prospective, placebo-controlled trial. BJU Int. 1999;84(6):622-627.
8. McNeill SA, Hargreave TB, Geffriaud-Ricouard C, Santoni JP, Roehrborn CG. Postvoid residual urine in patients with lower urinary tract symptoms suggestive of benign prostatic hyperplasia: pooled analysis of eleven controlled studies with Alfuzosin. Urology. 2001;57(3):459-465.

9. Debruyne F, Jardin A, Colloi D, Resel I, Witjes W, Delauche-Cavallier M, McCarthy C, Geffiaud-Ricouard C. Sustained-release Alfuzosin, Finasteride and the combination of both in the treatment of benign prostatic hyperplasia. Eur Urol. 1998;34 (3):169-175.

10. Nickel JC, Elhilali M, Vallancien G; ALF-ONE Study Group. Benign prostatic hyperplasia $(\mathrm{BPH})$ and prostatitis: prevalence of painful ejaculation in men with clinical BPH. BJU Int. 2005;95(4):571-574.

11. Clifford GM, Farmer RD. Medical therapy for benign prostatic hyperplasia. A review of the literature. Eurol. Urol. 2000;38(1): 2-19.

12. Kirby RS, O`Leary MP, Carson C. Efficacy of extended-release doxazosin and doxazosin standard in patients with concomitant benign prostatic hyperplasia and sexual dysfunction. BJU Int. 2005;95(1): 103-109.

13. Badía X, Baró E. Cuestionario de salud en España y su uso en Atención Primaria. Atención Primaria. 2001;5(28):349-356.

14. Rosen RC, Catania J, Pollack L, Althof S, O`Leary M, Seftel AD. Male sexual health questionnaire (MSHQ): scale development and psychometric validation. Urology. 2004;64(4):777-782.

15. Rosen RC. Update of the relationship between sexual dysfunction and lower urinary tract symptoms/benign prostatic hyperplasia. Curr Opin Urol. 2006;16(1):11-19.

16. Rosen R, Altwein J, Boyle P, Kirby RS, Lukacs B, Meuleman E, et al. Lower urinary tract symptoms and male sexual dysfunction: the multinational survey of the aging male (MSAM-7). Eur Urol. 2003;44(6):637-649.

17. Nickel JC, Elhilali M, Emberton M, Vallancien G; ALF-ONE Study Group. The beneficial effect of alfuzosin $10 \mathrm{mg}$ once daily in "real-life" practice on lower urinary tract symptoms (LUTS), quality of life and sexual dysfunction in men with LUTS and painful ejaculation. BJU Int. 2006;97(6):1242-1246.

18. Hellstrom WJ, Sikka SC. Effects of acute treatment with tamsulosin versus alfuzosin on ejaculatory function in normal volunteers. J Urol. 2006;176(4Pt1):1529-1533.

Correspondencia autor: Dr. A. Martín Morales

Servicio de Urología, Unidad de Andrología.

Hospital Carlos Haya. Plaza Hospital Civil, s/n - 29009 Málaga

Tel.: 951290362

E-mail autor: amartinmorales@terra.es

Información artículo: Original - HBP

Trabajo recibido: diciembre 2007

Trabajo aceptado: abril 2008 